\title{
Neuro(re)development of brain circuitry: linking cell biology to psychiatric discoveries
}

\author{
B. lan Hutchins* \\ Cellular and Developmental Neurobiology Section, National Institute of Neurological Disorders and Stroke, Bethesda, MD, USA \\ ${ }^{*}$ Correspondence: bruce.hutchins@nih.gov \\ Edited by: \\ Ahmet O. Caglayan, Yale University, USA \\ Reviewed by: \\ Kenneth Yu-Chung Kwan, Yale School of Medicine, USA \\ Angela Heinrich, Heidelberg University, Germany \\ Reto Bisaz, New York University, USA
}

\section{A commentary on}

Identification of risk loci with shared effects on five major psychiatric disorders: a genome-wide analysis

by Cross-Disorder Group of the Psychiatric Genomics Consortium, Smoller JW, Craddock $N$, Kendler K, Lee PH, Neale BM, Nurnberger JI, Ripke S, Santangelo S, Sullivan PF. Lancet (2013) 381(9875):1371-9. doi: 10.1016/ S0140-6736(12)62129-1

Neurodevelopmental defects are thought to contribute to psychiatric disorders by disrupting the proper wiring of neural circuits. While these defects may underlie putative developmental disorders like schizophrenia, their contribution to others, like major depressive disorder, are less clear. Genome-wide association studies (GWAS) have identified many genes associated with individual disorders. However, given the diverse etiologies and ages of onset of common psychiatric disorders, one might expect individual gene variants to associate mainly with one or two disorders sharing common mechanisms (e.g., defects in synaptic transmission or disrupted axon connectivity). It was therefore surprising that the Cross-Disorder Group of the Psychiatric Genomics Consortium recently identified mutations in L-type calcium channels common to five psychiatric disorders (1), not all of which are thought to be neurodevelopmental in origin (autism spectrum disorder, attention deficit-hyperactivity disorder, bipolar disorder, major depressive disorder, and schizophrenia).

One possibility raised by these results is that a fundamental mechanism is used by the nervous system throughout early development and into adulthood, that, when disrupted, increases susceptibility to psychiatric disorders in general. If there were such a fundamental mechanism at work, what would it look like? First, it would of course have to operate through L-type channels. Second, it would have to be utilized broadly in the nervous system to account for the pleiotropic effects of these mutations. Third, it would have to operate both during neurodevelopment and in mature circuitry, since the Psychiatric Genomics Consortium (1) implicated these genes in disorders of varied neurodevelopmental contributions.

Basic research from the last decade has identified a common cell biological mechanism that meets these criteria and could potentially explain this source of shared risk for psychiatric disorders: competitive axon remodeling. During competitive axon remodeling, electrical activity drives a local depolarization that leads to L-type voltage gated calcium channel activation and calcium influx localized to a branch or small group of branches (2). These stimulated branches are favored for extension or further branching, and a competitive mechanism is engaged that causes pruning of unstimulated branches from the same axon (2-4). Blocking L-type calcium channels slows competitive axon remodeling, while stimulating these channels accelerates this process (2), fulfilling the first criterion of dependence on L-type channels. An example of this process is shown in Figure 1. This process has been identified in cortical neurons (2), hippocampal neurons (3), and peripheral neurons (4), meeting the second criterion of broad utilization. Finally, imaging of axon dynamics in the adult neocortex has demonstrated that mature axons continue to dynamically extend and retract branches $(5,6)$. Although at a lower rate than in pre- and post-natal critical periods, this process still occurs at these later, post-developmental stages of life. In addition to the large-scale axon remodeling in development, the capacity for experiencedependent, competitive axon remodeling is preserved through adulthood in primates as well $(7,8)$. This shows that axon remodeling is not limited to developmental windows, but instead that neural circuits continue to "re-develop" with experience in adulthood.

Importantly, gene expression data from humans (Human Brain Transcriptome) suggest that the two L-type calcium channelassociated genes identified by the Psychiatric Genomics Consortium (CACNA1C and CACNB2) (1) are broadly expressed in the nervous system. Furthermore, expression of these genes is upregulated between embryonic weeks 13-19, when long-distance axonal tracts such as the corpus callosum are being established (9). Unlike some developmental genes, the expression of these two genes remains high through adulthood. This places these genes at the right times and in a sufficiently broad distribution of brain areas to influence competitive axon remodeling in each of these psychiatric disorders.

Continued axon remodeling throughout life provides a conceptual framework of neurodevelopment (and the slower neural re-development in adulthood) that could go awry during psychiatric illness. As with any proposed mechanism relating the molecular genetics with the observed clinical outcomes, empirical validation, or refutation is required. Validation could yield important alternative signaling pathways that influence competitive axon growth (10). In addition, this type of investigation is more likely 


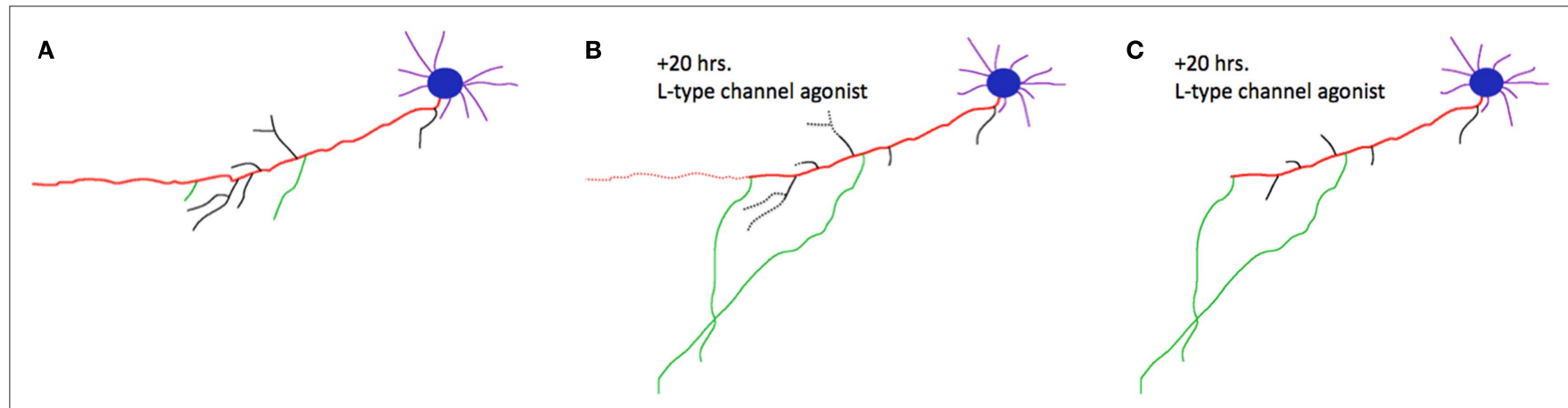

FIGURE 1 | Competitive axon remodeling accelerated by an L-type calcium channel agonist. (A) Tracing of a cortical neuron grown in dissociated tissue culture. The primary axon is shown in red; branches are shown in black, or green for emphasis. Blue, cell body; purple, dendrites. (B) After $20 \mathrm{~h}$ in a L-type calcium channel agonist, the primary axon, and many branches dramatically retracted (retractions shown with dotted lines). The green branches were favored for extension in this competitive process; dendrites were largely unaffected. (C) Final morphology of the neuron after $20 \mathrm{~h}$. Figure adapted from (2). to elucidate the biological underpinnings of disease, which the National Institute of Mental Health recently endorsed in favor of symptom-based diagnosis used in the Diagnostic and Statistical Manual of Mental Health. Although it is not currently clear that a single common mechanism links L-type channel mutations to the associated disorders, pursuit of this kind of fundamental link is worthwhile for the conceptual advances and therapeutic targets that might be discovered if true.

\section{ACKNOWLEDGMENTS}

Bruce Ian Hutchins is supported by the Intramural Research Program of NINDS and the Postdoctoral Research Associate Program of NIGMS. I thank Dr. Ellen Flannery for thoughtful comments on the manuscript.

\section{REFERENCES}

1. Cross-Disorder Group of the Psychiatric Genomics Consortium, Smoller JW, Craddock N, Kendler K, Lee PH, Neale BM, et al. Identification of risk loci with shared effects on five major psychiatric disorders: a genome-wide analysis. Lancet (2013) 381(9875):1371-9. doi: 10.1016/ S0140-6736(12)62129-1

2. Hutchins BI, Kalil K. Differential outgrowth of axons and their branches is regulated by localized calcium transients. J Neurosci (2008) 28 (1):143-53. doi: 10.1523/JNEUROSCI.4548-07.2008

3. Lee H, Lee D, Park CH, Ho WK, Lee SH. GABA mediates the network activity-dependent facilitation of axonal outgrowth from the newborn granule cells in the early postnatal rat hippocampus. Eur J Neurosci (2012) 36(6):2743-52. doi: 10.1111/j.1460-9568.2012.08192.x

4. Singh KK, Miller FD. Activity regulates positive and negative neurotrophin-derived signals to determine axon competition. Neuron (2005) 45(6):837-45. doi: 10.1016/j.neuron.2005.01.049

5. De PaolaV, Holtmaat A, Knott G, Song S, Wilbrecht $\mathrm{L}$, Caroni P, et al. Cell type-specific structural plasticity of axonal branches and boutons in the adult neocortex. Neuron (2006) 49(6):861-75. doi: 10.1016/j. neuron.2006.02.017

6. Stettler DD, Yamahachi H, Li W, Denk W, Gilbert CD. Axons and synaptic boutons are highly dynamic in adult visual cortex. Neuron (2006) 49(6):877-87. doi: 10.1016/j.neuron.2006.02.018

7. Gilbert CD, Li W. Adult visual cortical plasticity. Neuron (2012) 75(2):250-64. doi: 10.1016/j. neuron.2012.06.030
8. Yamahachi H, Marik SA, McManus JN, Denk W, Gilbert CD. Rapid axonal sprouting and pruning accompany functional reorganization in primary visual cortex. Neuron (2009) 64(5):719-29. doi: 10.1016/j.neuron.2009.11.026

9. Ren T, Anderson A, Shen WB, Huang H, Plachez C, Zhang J, et al. Imaging, anatomical, and molecular analysis of callosal formation in the developing human fetal brain. Anat Rec (2006) 288A:191-204. doi: 10.1002/ar.a.20282

10. Shelly M, Lim BK, Cancedda L, Heilshorn SC, Gao H, Poo MM. Local and long-range reciprocal regulation of cAMP and cGMP in axon/dendrite formation. Science (2010) 327(5965):547-52. doi: 10.1126/science. 1179735

Received:04 June 2013; accepted: 23 June 2013; published online: 08 July 2013.

Citation: Hutchins BI (2013) Neuro(re)development of brain circuitry: linking cell biology to psychiatric discoveries. Front. Psychiatry 4:65. doi: 10.3389/fpsyt.2013.00065 This article was submitted to Frontiers in Child and Neurodevelopmental Psychiatry, a specialty of Frontiers in Psychiatry.

Copyright (C) 2013 Hutchins. This is an open-access article distributed under the terms of the Creative Commons Attribution License, which permits use, distribution and reproduction in other forums, provided the original authors and source are credited and subject to any copyright notices concerning any third-party graphics etc. 